PROCEEDINGS OF THE

AMERICAN MATHEMATICAL SOCIETY

Volume 136, Number 8, August 2008, Pages 2823-2828

S 0002-9939(08)09561-0

Article electronically published on April 15, 2008

\title{
A NEW MAXIMUM PRINCIPLE OF ELLIPTIC DIFFERENTIAL EQUATIONS IN DIVERGENCE FORM
}

\author{
DONGSHENG LI AND LIHE WANG \\ (Communicated by David S. Tartakoff)
}

\begin{abstract}
In this paper will be presented a new maximum principle of elliptic differential equations in divergence form which can be regarded as the counterpart of the Alexandroff-Bakelman-Pucci maximum principle of elliptic differential equations in nondivergence form.
\end{abstract}

\section{INTRODUCTION}

The Alexandroff-Bakelman-Pucci maximum principle is an important tool for studying elliptic differential equations in nondivergence form. In this paper, we shall find the counterpart of it for elliptic differential equations in divergence form.

Throughout this paper, we assume that $\Omega \subset R^{n}$ is a bounded domain, $f(x) \in$ $L^{p}(\Omega)$ and $a^{i j}(x) \in L^{\infty}(\Omega), i, j=1,2, \ldots, n$ such that for $x \in \Omega$ a.e., $\left(a^{i j}\right)_{n \times n}$ is symmetric and

$$
\lambda I \leq\left(a^{i j}\right)_{n \times n} \leq \Lambda I
$$

in the sense of nonnegative definiteness, where $\lambda \leq \Lambda$ are two positive constants. We denote by $B_{r}$ the open ball in $R^{n}$ centered at the origin with radius $r$. For convenience, we extend $\left(a^{i j}(x)\right)_{n \times n}$ to be the identity outside $\Omega$ and still denote the extended matrix by $\left(a^{i j}(x)\right)_{n \times n}$. Since $\Omega$ is bounded, we assume that

$$
\Omega \subset B_{d}
$$

for $d>0$. Let $\chi_{E}$ denote the character function of the set $E$, and define $f^{+}=$ $\max \{0, f\}$ and $f^{-}=\max \{0,-f\}$ for any function $f$.

To compare with our new maximum principle, we first state our AlexandroffBakelman-Pucci maximum principle as the following (cf. [1, 2] for the proof).

Theorem 1.1 (Alexandroff-Bakelman-Pucci maximum principle). Suppose $f \in$ $L^{p}(\Omega)$ and $u \in W^{2, p}(\Omega)$ is a subsolution of the following:

$$
-a^{i j}(x) \partial_{i j} u(x)=f(x)
$$

Received by the editors August 1, 2005, and, in revised form, January 20, 2007.

2000 Mathematics Subject Classification. Primary 35J25.

Key words and phrases. Maximum principle, elliptic equation in divergence form.

The first author was supported by the NSF of China: 10771166.

The second author was supported by PCSIRT.

(C)2008 American Mathematical Society Reverts to public domain 28 years from publication 
in $\Omega$. If $p \geq n$, then there exists a constant $C$ depending only on $\lambda, \Lambda, p$ and $n$ such that

$$
\sup _{\Omega} u \leq \sup _{\partial \Omega} u^{+}+C \cdot d^{2-\frac{n}{p}} \cdot\left(\int_{\left\{\psi=\Gamma_{\psi}\right\} \cap \Omega}\left(f^{+}\right)^{p}\right)^{\frac{1}{p}}
$$

where $\psi=u-\sup _{\partial \Omega} u^{+}$and $\Gamma_{\psi}$ is the concave envelope of $\psi$ in $B_{2 d}$ (cf. 1] for its definition).

For the following elliptic differential equation in divergence form,

$$
-\partial_{i}\left(a^{i j}(x) \partial_{j} u(x)\right)=f(x) \text { in } \Omega,
$$

the estimate of (1.3) cannot be obtained by exploiting the concave envelope $\Gamma_{\psi}$ as Theorem 1.1 since the concave envelope is appropriate for (1.2) while not for (1.4). However, if we replace the concave envelope by another proper function, a similar estimate of (1.3) can still be obtained. In fact, the proper function is the solution of the following obstacle problem:

$$
\left\{\begin{array}{l}
\text { Find } u \in K_{\psi} \text { such that } \\
J(u)=\inf _{w \in K_{\psi}} J(w),
\end{array}\right.
$$

where

$$
J(w)=\int_{B_{2 d}} a^{i j} \partial_{i} w \partial_{j} w
$$

for any $w \in W_{0}^{1,2}\left(B_{2 d}\right)$ and

$$
K_{\psi}=\left\{w \in W_{0}^{1,2}\left(B_{2 d}\right): w \geq \psi \text { a.e. in } \Omega\right\}
$$

for any fixed $\psi \in W^{1,2}(\Omega)$ with $\psi \leq 0$ on $\partial \Omega$. For Problem (1.5), we have the following well-known results (cf. 3], [4]):

Lemma 1.2. (i) There exists a unique $\Upsilon_{\psi} \in K_{\psi}$ such that

$$
J\left(\Upsilon_{\psi}\right)=\inf _{w \in K_{\psi}} J(w) .
$$

(ii) There exists a unique $\tilde{\Upsilon}_{\psi} \in K_{\psi}$ such that

$$
\int_{B_{2 d}} a^{i j} \partial_{i} w \partial_{j}\left(w-\tilde{\Upsilon}_{\psi}\right) \geq 0, \quad \forall w \in K_{\psi} .
$$

(iii) There exists a unique $\hat{\Upsilon}_{\psi} \in K_{\psi}$ such that

$$
\int_{B_{2 d}} a^{i j} \partial_{i} \hat{\Upsilon}_{\psi} \partial_{j}\left(w-\hat{\Upsilon}_{\psi}\right) \geq 0, \quad \forall w \in K_{\psi} .
$$

(iv) $\Upsilon_{\psi}=\tilde{\Upsilon}_{\psi}=\hat{\Upsilon}_{\psi}$.

Now we can present our maximum principle for (1.4) as the following:

Theorem 1.3 (Maximum Principle). Suppose $f \in L^{p}(\Omega)$ with $p>\frac{n}{2}$ if $n \geq 4$ or $p=2$ if $n=1,2,3$, and $u \in W^{1,2}(\Omega)$ is a subsolution of (1.4). Then there is a constant $C$ depending only on $\lambda, n$ and $p$ such that

$$
\sup _{\Omega} u \leq \sup _{\partial \Omega} u^{+}+C \cdot d^{2-\frac{n}{p}} \cdot\left(\int_{\left\{\psi=\Upsilon_{\psi}\right\} \cap \Omega}\left(f^{+}\right)^{p}\right)^{\frac{1}{p}},
$$

where $\psi=u-\sup _{\partial \Omega} u^{+}$and $\Upsilon_{\psi}$ is given by Lemma 1.2 . 
In section 2, we study the regularity of the solution of the obstacle problem (1.5). In section 3 , we shall prove our main result, Theorem 1.3.

\section{Regularity of SOlutions of OBStaCle PROBlems}

Our regularity result for the obstacle problem (1.5) is the following:

Theorem 2.1. Suppose $f \in L^{2}(\Omega)$ and $\psi \in W^{1,2}(\Omega)$ with $\psi \leq 0$ on $\partial \Omega$ such that

$$
-\partial_{i}\left(a^{i j} \partial_{j} \psi\right) \leq f
$$

in the weak sense in $\Omega$. Then we have

$$
0 \leq-\partial_{i}\left(a^{i j}(x) \partial_{j} \Upsilon_{\psi}(x)\right) \leq f^{+}(x) \cdot \chi_{E}
$$

in the weak sense in $B_{2 d}$, where $\Upsilon_{\psi}$ is given by Lemma 1.2 and $E=\{x \in \Omega$ : $\left.\psi(x)=\Upsilon_{\psi}(x)\right\}$.

If $a^{i j}, f$ and $\psi$ are smooth and $-\partial_{i}\left(a^{i j} \partial_{j} \psi\right)=f$ in $\Omega$, Lewy and Stampacchia [3] showed that

$$
-\partial_{i}\left(a^{i j}(x) \partial_{j} \Upsilon_{\psi}(x)\right)=f^{+}(x) \cdot \chi_{E}
$$

for $x \in B_{2 d}$ a.e. The idea used in $[3$ with some appropriate changes is also applicable for the proof of Theorem 2.1. The first part of (2.2) is well known (cf. 3], 4]), and then we only need to show the second part of it, which needs the following two lemmas.

Lemma 2.2. Suppose $w \in W^{1,2}(\Omega)$ such that $w \geq 0$ on $\partial \Omega$ and

$$
-\partial_{i}\left(a^{i j}(x) \partial_{j} w(x)\right) \geq f(x)
$$

in the weak sense in $\Omega$, where $f \in L^{2}(\Omega)$. If $w(x) \leq 0$ implies $f(x) \geq 0$ for $x \in \Omega$ a.e., then we have $w \geq 0$ a.e. in $\Omega$.

Proof. Since $w(x) \geq 0$ on $\partial \Omega$, we have $w^{-} \in W_{0}^{1,2}(\Omega)$. From (2.3) and the assumption that $w(x) \leq 0$ implies $f(x) \geq 0$ for $x \in \Omega$ a.e., it follows that

$$
\int_{\Omega} a^{i j} \partial_{i} w \partial_{j} w^{-} \geq \int_{\Omega} f w^{-}=\int_{E} f w^{-} \geq 0
$$

where $E=\{x \in \Omega: w(x) \leq 0\}$. Thereby,

$$
\lambda \int_{\Omega}\left|D w^{-}\right|^{2} \leq \int_{\Omega} a^{i j} \partial_{i} w^{-} \partial_{j} w^{-}=\int_{\Omega} a^{i j} \partial_{i}(-w) \partial_{j} w^{-} \leq 0 .
$$

Hence $w^{-}=0$ a.e. in $\Omega$.

Lemma 2.3. Suppose $f \in L^{2}(\Omega)$ and $\theta$ is a Lipschitz function defined in $(-\infty,+\infty)$ such that $0 \leq \theta \leq 1$. Then the nonlinear equation

$$
-\partial_{i}\left(a^{i j}(x) \partial_{j} u(x)\right)=f^{+}(x) \cdot \theta(u-\psi) \cdot \chi_{\Omega}
$$

has a solution in $W_{0}^{1,2}\left(B_{2 d}\right)$ for any $\psi \in L^{2}(\Omega)$. 
Proof. We use the Schauder fixed point theory. For any $w \in L^{2}\left(B_{2 d}\right)$, the equation

$$
-\partial_{i}\left(a^{i j}(x) \partial_{j} u(x)\right)=f^{+}(x) \cdot \theta(w-\psi) \cdot \chi_{\Omega}
$$

has a unique weak solution $u \in W_{0}^{1,2}\left(B_{2 d}\right)$, and we denote $u=T w$. Since $0 \leq \theta \leq 1$, there exists a constant $C$ depending only on $\lambda$ and $d$ such that

$$
\|T w\|_{W^{1,2}\left(B_{2 d}\right)} \leq C\left\|f^{+}\right\|_{L^{2}(\Omega)}, \quad \forall w \in L^{2}\left(B_{2 d}\right) .
$$

Thereby the compactness of $T$ from $L^{2}\left(B_{2 d}\right)$ to $L^{2}\left(B_{2 d}\right)$ follows clearly. If we set $D=\left\{w \in L^{2}\left(B_{2 d}\right):\|w\|_{L^{2}\left(B_{2 d}\right)} \leq C\left\|f^{+}\right\|_{L^{2}(\Omega)}\right\}$, then $T(D) \subset T\left(L^{2}\left(B_{2 d}\right)\right) \subset D$. Since $\theta$ is Lipschitz continuous, $T$ is continuous from $L^{2}\left(B_{2 d}\right)$ to $W_{0}^{1,2}\left(B_{2 d}\right)$ and then to $L^{2}\left(B_{2 d}\right)$. Therefore, according to the Schauder fixed point theory, there exists $u \in D$ such that $u=T u \in W_{0}^{1,2}\left(B_{2 d}\right)$.

Proof of Theorem 2.1. Consider a sequence of functions $\left\{\theta_{m}\right\}_{m=1}^{\infty}$ defined by

$$
\theta_{m}= \begin{cases}1 & \text { for } \quad t \leq \frac{1}{2 m}, \\ 2-2 m t & \text { for } \quad \frac{1}{2 m} \leq t \leq \frac{1}{m}, \\ 0 & \text { for } \quad t \geq \frac{1}{m},\end{cases}
$$

and let $u_{m} \in W_{0}^{1,2}\left(B_{2 d}\right)$ be a solution of (2.4) with $\theta=\theta_{m}$.

We first claim that $u_{m} \geq \psi$ a.e. in $\Omega$ for any $m$. Indeed, the maximum principle implies $u_{m} \geq 0$ a.e. in $B_{2 d}$. In view of $\psi \leq 0$ on $\partial \Omega$, we deduce $u_{m}-\psi \geq 0$ on $\partial \Omega$. By (2.1) and (2.4) with $\theta$ and $u$ replaced by $\theta_{m}$ and $u_{m}$, respectively,

$$
-\partial_{i}\left(a^{i j} \partial_{j}\left(u_{m}-\psi\right)\right) \geq f^{+}(x) \cdot \theta_{m}\left(u_{m}-\psi\right)-f
$$

in the weak sense in $\Omega$. Since $\left(f^{+} \cdot \theta_{m}\left(u_{m}-\psi\right)-f\right)(x) \geq 0$ if $\left(u_{m}-\psi\right)(x) \leq 0$ for $x \in \Omega$ a.e., by Lemma 2.2, our claim is proved.

It is clear that $\left\{u_{m}\right\}$ is bounded in $W_{0}^{1,2}\left(B_{2 d}\right)$. Without loss of generality, we assume that

$$
u_{m} \rightarrow \tilde{u} \text { weakly in } W_{0}^{1,2}\left(B_{2 d}\right) \text {, strongly in } L^{2}\left(B_{2 d}\right) \text { and a.e. in } B_{2 d} .
$$

We let $\tilde{E}=\{x \in \Omega: \tilde{u}(x)=\psi(x)\}$ and claim that

$$
\limsup _{m \rightarrow \infty} \theta_{m}\left(u_{m}-\psi\right) \leq \chi_{\tilde{E}} \text { a.e. in } \Omega .
$$

In fact, by (2.6) and $u_{m} \geq \psi$ a.e. in $\Omega$, there exists $K \subset \Omega$ with $|K|=0$ such that $u_{m}(x) \rightarrow \tilde{u}(x)$ and $u_{m}(x) \geq \psi(x)$ for all $x \in \Omega \backslash K$. If $x \in \tilde{E}$, then (2.7) holds clearly since $\theta_{m} \leq 1$. Suppose $x \in \Omega \backslash\{\tilde{E} \cup K\}$ and then $\tilde{u}(x)>\psi(x)$. Since $u_{m}(x) \rightarrow \tilde{u}(x)$, there exists $m_{0}$ such that if $m \geq m_{0}, u_{m}(x)-\psi(x)>\frac{1}{m}$. Thereby $\theta_{m}\left(u_{m}(x)-\psi(x)\right)=0$ for $m \geq m_{0}$. Hence, (2.7) holds.

Since for any $\phi \in C_{0}^{1}\left(B_{2 d}\right)$ with $\phi \geq 0$,

$$
\int_{B_{2 d}} a^{i j} \partial_{i} u_{m} \partial_{j} \phi=\int_{B_{2 d}} f^{+} \cdot \theta_{m}\left(u_{m}-\psi\right) \cdot \chi_{\Omega} \cdot \phi,
$$

by taking the limit in both sides and in view of $(2.6),(2.7)$, we deduce

$$
\int_{B_{2 d}} a^{i j} \partial_{i} \tilde{u} \partial_{j} \phi \leq \int_{B_{2 d}} f^{+} \cdot \chi_{\tilde{E}} \cdot \phi
$$


If we can show that $\tilde{u}=\Upsilon_{\psi}$, then the proof of Theorem 2.1 will be complete. Indeed, for each $m$, since $\theta_{m}$ is decreasing, we have for any $v \in W_{0}^{1,2}\left(B_{2 d}\right)$,

$$
\int_{B_{2 d}}\left\{a^{i j} \partial_{i}\left(v-u_{m}\right) \partial_{j}\left(v-u_{m}\right)-f^{+} \cdot\left(\theta_{m}(v-\psi)-\theta_{m}\left(u_{m}-\psi\right)\right) \cdot \chi_{\Omega} \cdot\left(v-u_{m}\right)\right\} \geq 0 .
$$

Combining with (2.4), where $\theta$ and $u$ are replaced by $\theta_{m}$ and $u_{m}$ respectively, we obtain

$$
\int_{B_{2 d}}\left\{a^{i j} \partial_{i} v \partial_{j}\left(v-u_{m}\right)-f^{+} \cdot \theta_{m}(v-\psi) \cdot \chi_{\Omega} \cdot\left(v-u_{m}\right)\right\} \geq 0 .
$$

For any fixed positive integer $m_{0}$, in (2.9), take $v-\psi>\frac{1}{m_{0}}$ a.e. in $\Omega$ and then

$$
\int_{B_{2 d}} a^{i j} \partial_{i} v \partial_{j}\left(v-u_{m}\right) \geq 0
$$

for $m>m_{0}$. By $(2.6)$,

$$
\int_{B_{2 d}} a^{i j} \partial_{i} v \partial_{j}(v-\tilde{u}) \geq 0 .
$$

Since $m_{0}$ is arbitrary, we have that (2.10) holds for any $v \in K_{\psi}$. Then Lemma 1.2 implies $\tilde{u}=\Upsilon_{\psi}$.

\section{Proof of Theorem 1.3}

To prove Theorem 1.3, we also need the following theorem, which can be proved by Moser iteration (cf. [2]) or by the rearrangement technique (cf. [5]).

Theorem 3.1. Suppose $f \in L^{p}(\Omega)$ with $p>\frac{n}{2}$ and $u \in W^{1,2}(\Omega)$ is a subsolution of (1.4). Then there is a constant $C$ depending only on $\lambda, n$ and $p$ such that

$$
\sup _{\Omega} u \leq \sup _{\partial \Omega} u^{+}+C \cdot r^{2-\frac{n}{p}} \cdot\|f\|_{L^{p}(\Omega)},
$$

where $r$ satisfies $|\Omega|=\omega_{n} r^{n}$ and $\omega_{n}$ denotes the volume of the unit ball in $R^{n}$.

Remark 3.2. It is clear that the estimate of $u$ by Theorem 1.3 is more accurate than by Theorem 3.1 .

Proof of Theorem 1.3. Set $\psi=u-\sup _{\partial \Omega} u^{+}$, and then (2.1) holds. Let $\Upsilon_{\psi}$ be given by Lemma 1.2. From Theorem 2.1, it follows that

$$
-\partial_{i}\left(a^{i j} \partial_{j} \Upsilon_{\psi}\right) \leq f^{+} \cdot \chi_{\left\{\psi=\Upsilon_{\psi}\right\} \cap \Omega}
$$

in the weak sense in $B_{2 d}$. In view of Theorem 3.1, there exists a constant $C$ depending only on $\lambda, n$ and $p$ such that

$$
\sup _{\Omega} \Upsilon_{\psi} \leq C \cdot d^{2-\frac{n}{p}} \cdot\left\|f^{+} \cdot \chi_{\left\{\psi=\Upsilon_{\psi}\right\} \cap \Omega}\right\|_{L^{p}(\Omega)} .
$$

Combining with

$$
\sup _{\Omega} u-\sup _{\partial \Omega} u^{+} \leq \sup _{\Omega} \psi \leq \sup _{\Omega} \Upsilon_{\psi},
$$

we have that (1.6) holds. 


\section{REFERENCES}

[1] Caffarelli, L. A., and Cabre, X., Fully nonlinear elliptic equations, Colloquium Publications, 43, Amer. Math. Soc., Providence, RI, 1995. MR.1351007 (96h:35046)

[2] Gilbarg, D., and Trudinger, N. S., Elliptic partial differential equations of second order, 2nd ed., Springer-Verlag, 1983. MR737190 (86c:35035)

[3] Lewy, H., and Stampacchia, G., On the regularity of the solution of a variational inequality, Comm. on Pure and Appl. Math., 1969, XXII, 153-188. MR0247551 (40:816)

[4] Lions, J.-L., and Stampacchia, G., Variational inequalities, Comm. on Pure and Appl. Math., 1967, XX, 493-519. MR0216344 (35:7178)

[5] Talenti, G., Elliptic equations and rearrangements, Annali della Scuola Norm. Sup. di Pisa, 1976, 3, 697-718. MR0601601(58:29170)

College of Science, Xi'an Jiaotong University, Xi'an 710049, China

E-mail address: lidsh@mail.xjtu.edu.cn

Department of Mathematics, The University of Iowa, Iowa City, Iowa 52242-1419

E-mail address: lwang@math.uiowa.edu 